\title{
Local Aspects of Superselection Rules. II^
}

\author{
Sergio Doplicher and Roberto Longo \\ Istituto Matematico G. Castelnuovo, Università di Roma, I-00185 Roma, Italy
}

\begin{abstract}
In a theory where the local observables are determined by local field algebras as the fixed points under a (a priori noncommutative) group of gauge transformations of the first kind, we show that, if the field algebras possess intermediate type I factors, we can construct observables having the meaning of local charge measurements, and local current algebras in the field algebras.
\end{abstract}

\section{Introduction}

In this paper we deal with the problem of proving, in a local quantum theory, the existence of observables associated to a given compact region in space-time which measure the superselection quantum numbers ("charges") contained in a smaller region. In [1] this problem was studied for a theory obeying two kinds of restrictions. Firstly, the charges should produce no long range correlations, i.e. the superselection structure should correspond to the "states of interest" of the theory studied in [2]. Second there should be no parastatistics; in addition to these, there were other restrictions made there, which however correspond to properties one would expect to hold in physically meaningful models.

In the discussion of [1], to which we refer for the motivations, the problem was formulated in terms of structural properties of the algebras of observable quantities alone. Here we remove the second type of restrictions, i.e. we deal with local quantum theories with a compact group of internal symmetry (the group of gauge transformations of the first kind) which is possibly nonabelian. This case differs from case [1] in two respects. First, in the presence of parastatistics, there is no theorem of existence and uniqueness of a field algebra obeying normal commutation relations associated to the net of local observables (see, however, $[2,3])$. Second, the densities for the generators of the gauge transformations, which are the ultimate unknown of our problem, are not gauge invariant, i.e. cannot be observables. Therefore we limit ourselves to the case where the net of local algebras of field operators is given as the primary object.

* Research supported by Minî́stero della Pubblica Istruzione and C.N.R.-G.n.a.fa. 
Namely, we assume that there is a correspondence

$$
\mathcal{O} \rightarrow \mathfrak{F}(\mathcal{O})
$$

between double cones in Minkowski space and von Neumann algebras on a fixed Hilbert space $\mathscr{H}$, and a unitary continuous representation $\mathscr{U}$ of a compact group $\mathscr{G}$ on $\mathscr{H}$ so that

$$
\mathscr{U}(g) \mathfrak{F}(\mathcal{O}) \mathscr{U}(g)^{-1}=\mathfrak{F}(\mathcal{O}), \quad g \in \mathscr{G}, \quad \mathcal{O} \in \mathscr{K},
$$

where $\mathscr{K}$ denotes the set of all double cones in Minkowski space. Then $\mathscr{F}(\mathcal{O})$ is interpreted as the algebra generated by the field operators localized in $\mathcal{O}$ and the automorphisms $\alpha_{g}$ induced by $\mathscr{U}(g)$ on each $\mathfrak{F}(\mathcal{O}), g \in \mathscr{G}$, as the gauge automorphisms. The subalgebra $\mathfrak{A}(\mathcal{O})$ of the gauge invariant elements in $\mathfrak{F}(\mathcal{O})$ is the algebra of observables localized in $\mathcal{O}$.

For each pair $\mathcal{O}_{1}, \mathcal{O}_{2} \in \mathscr{K}$ such that $\mathcal{O}_{1} \ll \mathcal{O}_{2}$ in the sense that $\mathcal{O}_{1}$ is contained in the interior of $\mathscr{O}_{2}$, we want to find a unitary continuous representation $\mathscr{V}$ of $\mathscr{G}$ on $\mathscr{H}$, such that

$$
\begin{gathered}
\mathscr{V}_{g} \in \mathfrak{F}\left(\mathcal{O}_{2}\right), \quad \mathscr{V}_{g} F \mathscr{V}_{g}^{-1}=\alpha_{g}(F), \quad F \in \mathfrak{F}\left(\mathcal{O}_{1}\right), \\
\alpha_{h}\left(\mathscr{V}_{g}\right)=\mathscr{V}_{h g h^{-1}}, \quad h, g \in \mathscr{G} .
\end{gathered}
$$

If $\mathscr{G}$ is abelian (1.4) reduces to the condition $\mathscr{V}_{g} \in \mathfrak{U}\left(\mathcal{O}_{2}\right)$ as in [1]. We will show that under the Assumptions (i)-(iii) below this problem has a solution. It follows that the center $\mathscr{A}$ of the von Neumann algebra generated by the representation $\mathscr{V}$ belongs to the relative commutant $\mathfrak{U}\left(\mathcal{O}_{1}\right)^{\prime} \cap \mathfrak{H}\left(\mathcal{O}_{2}\right)$ and provides an algebra of commuting observables whose simultaneous measurement determines, in the sense discussed in [1], the superselection quantum numbers in $\mathcal{O}_{1}$ by an observation performed in $\mathrm{O}_{2}$.

In the case where $\mathscr{G}$ is a Lie group, the generators of the representation $\mathscr{V}$ provide a "local current algebra" [see Eqs. (2.9)]; we briefly discuss the relation of this structure to the current algebra hypothesis in particle physics.

We will need only a few of the assumptions characteristic of field theory [a central role is played instead by the technical assumption (iii) below], namely:

(i) If $\mathcal{O}_{1} \subset \mathcal{O}_{2}, \mathfrak{F}\left(\mathcal{O}_{1}\right) \subset \mathfrak{F}\left(\mathcal{O}_{2}\right) ; \mathcal{O}_{1}, \mathcal{O}_{2} \in \mathscr{K}$.

(ii) There is a unit vector $\Omega \in \mathscr{H}$ invariant under $\mathscr{U}$ :

$$
\mathscr{U}(g) \Omega=\Omega, \quad g \in \mathscr{G},
$$

which is cyclic and separating for each $\mathfrak{F}(\mathcal{O}), \mathcal{O} \in \mathscr{K}$ and, if $\mathcal{O}_{1} \in \mathcal{O}_{2}, \mathcal{O}_{1}, \mathcal{O}_{2} \in \mathscr{K}$, also for $\mathfrak{F}\left(\mathcal{O}_{1}\right)^{\prime} \cap \mathfrak{F}\left(\mathcal{O}_{2}\right)$.

(iii) For each pair of double cones such that $\mathcal{O}_{1} \ll \mathcal{O}_{2}$, there exists a type I factor $\mathfrak{N}$ such that

$$
\mathfrak{F}\left(\mathcal{O}_{1}\right) \subset \mathfrak{N} \subset \mathfrak{F}\left(\mathcal{O}_{2}\right) \text {. }
$$

Assumption (iii) is a property conjectured by Borchers and proved by Buchholz for the free field [4]; we refer to the comments in [1] and to the earlier literature $[5,6]$.

Assumption (ii) needs some comment. The state $\omega_{0}$ induced by $\Omega$ on the algebra of observables is the vacuum state. A well known consequence of the Reeh-Schlieder theorem in quantum field theory is that the vacuum is cyclic and separating for each $\mathfrak{F}(\mathcal{O})$. Our further assumption that $\Omega$ is also cyclic for 
$\mathfrak{F}\left(\mathcal{O}_{1}\right)^{\prime} \cap \mathfrak{F}\left(\mathcal{O}_{2}\right), \mathcal{O}_{1} \ll \mathcal{O}_{2}$, can be checked for theories having the more detailed structure described in [1] and obeying normal commutation relations. In this case we can apply the discussion of [1] to the model where the Bose field operators are taken to be the observables and $\mathscr{G}=\left\{e, h_{0}\right\}, h_{0}^{2}=e$, with $\alpha_{h_{0}}(F)= \pm F$ iff $F$ is a Bose respectively Fermi field operator. By [1, Proposition 3.3], for each pair $\mathcal{O}_{1}, \mathcal{O}_{2} \in \mathscr{K}$, $\mathcal{O}_{1} \mathbb{C O}_{2}$, there is a unitary Bose operator $U \in \mathfrak{F}\left(\mathcal{O}_{2}\right)$ such that

$$
U F U^{-1}=\alpha_{h_{0}}(F), \quad F \in \mathfrak{F}\left(\mathcal{O}_{1}\right) .
$$

With $\mathcal{O}_{0} \in \mathscr{K}, \mathcal{O}_{0} \subset \mathcal{O}_{1}^{\prime} \cap \mathcal{O}_{2}$, define the locally twisted algebra $\mathfrak{F}^{\text {lt }}\left(\mathcal{O}_{0}\right)$ by

$$
\begin{aligned}
\mathfrak{F}^{\mathrm{lt}}\left(\mathcal{O}_{0}\right) & =\mathfrak{F}_{+}\left(\mathcal{O}_{0}\right)+U \mathfrak{F}_{-}\left(\mathcal{O}_{0}\right), \\
\mathfrak{F}_{ \pm}\left(\mathcal{O}_{0}\right) & =\frac{1}{2}\left(\mathrm{id} \pm \alpha_{h_{0}}\right)\left(\mathfrak{F}\left(\mathcal{O}_{0}\right)\right) .
\end{aligned}
$$

By (1.7) and (1.8) and by normalcy of the commutation properties at spacelike distances we have

$$
\mathfrak{F}^{\mathrm{lt}}\left(\mathcal{O}_{0}\right) \subset \mathfrak{F}\left(\mathcal{O}_{1}\right)^{\prime} \cap \mathfrak{F}\left(\mathcal{O}_{2}\right)
$$

and by (1.8) $\mathbb{F}^{\text {lt }}\left(\mathcal{O}_{0}\right)$ is cyclic on the vacuum $\Omega$.

Therefore in the case of normal commutation relations the usual assumptions of field theory together with assumption (iii) imply (ii).

Note that locality or local commutativity of observables with fields do not appear explicitly among our assumptions, aside for the motivations for (ii) discussed above. Assumption (iii) plays a central role instead, and it would be quite desirable to know whether it follows from more general properties which might be expected to hold in nontrivial theories.

It is an important open problem how the present formalism should be modified in order to be appropriate for more general theories, with topological charges which are possibly not strictly localizable [3], or for a noncovariant description of the charged sectors in gauge theories, QED in particular.

The results in this note depend upon some general consequence of the structure of a pair of von Neumann algebras expressed by Assumptions (i)-(iii); this structure will be studied in more detail in [7] and is analyzed in Sect. 3 only in its aspects basic to our present applications to field theory. An important fact in this connection is the existence of a canonical choice of the type I factor in Assumption (iii), Eq. (1.7). If a local quantum theory fulfills the analog for the net of observables of Assumption (iii), we can describe it in terms of "semilocal nets" of type I factors (see Sect. 4), although the local algebras of observables are never expected to be type I.

\section{Local Charges and Current Algebra}

We will denote by $\omega_{0}(F)=(\Omega, F \Omega)$ for any $F \in \mathfrak{B}(\mathscr{H})$ the vector state induced by $\Omega$, and by $\omega_{\xi}$ the vector state of $\mathfrak{B}(\mathscr{H})$ induced by $\xi \in \mathscr{H}$. By our Assumptions (i)-(iii), Sect. 1, if $\mathcal{O}_{1}, \mathcal{O}_{2} \in \mathscr{K}, \mathcal{O}_{1} \in \mathcal{O}_{2}$, there is a vector $\xi \in \mathscr{H}$ which induces a product state on $\mathfrak{F}\left(\mathcal{O}_{1}\right) \vee \mathfrak{F}\left(\mathcal{O}_{2}\right)^{\prime}$, and more specifically

$$
\begin{array}{ll}
\omega_{\xi}\left(F_{1} F_{2}\right)=\omega_{0}\left(F_{1}\right) \omega_{0}\left(F_{2}\right), \quad & F_{1} \in \mathfrak{F}\left(\mathcal{O}_{1}\right), \\
& F_{2} \in \mathfrak{F}\left(\mathcal{O}_{2}\right)^{\prime} .
\end{array}
$$


The key point for the discussion in this section is that the vector $\xi$ can be chosen to be cyclic for $\mathfrak{F}\left(\mathcal{O}_{1}\right) \vee \mathfrak{F}\left(\mathcal{O}_{2}\right)^{\prime}[4]$ and, as will be shown in the next section, (Lemma 3.3), such that

$$
\mathscr{U}(g) \xi=\xi, \quad g \in \mathscr{G} .
$$

By Assumption (iii), Sect. 1, we can define an action $\alpha^{1}$ of $\mathscr{G}$ by automorphisms of $\mathfrak{F}\left(\mathcal{O}_{1}\right) \vee \mathfrak{F}\left(\mathcal{O}_{2}\right)^{\prime}$ by setting

$$
\alpha_{g}^{1}\left(F_{1} F_{2}\right)=\alpha_{g}\left(F_{1}\right) F_{2} ; \quad F_{1} \in \mathfrak{F}\left(\mathcal{O}_{1}\right), F_{2} \in \mathfrak{F}\left(\mathcal{O}_{2}\right)^{\prime} .
$$

By (2.1) $\omega_{\xi}$ is $\alpha^{1}$-invariant and since $\xi$ is a cyclic vector we can define a continuous unitary representation $\mathscr{V}$ of $\mathscr{G}$ by setting

$$
\mathscr{V}_{g} F \xi=\alpha_{g}^{1}(F) \xi, \quad F \in \mathfrak{F}\left(\mathcal{O}_{1}\right) \vee \mathfrak{F}\left(\mathcal{O}_{2}\right)^{\prime} .
$$

Then by (2.3) $\mathscr{V}_{g}$ induces $\alpha_{g}$ on $\mathfrak{F}\left(\mathcal{O}_{1}\right)$ and commutes with $\mathfrak{F}\left(\mathcal{O}_{2}\right)^{\prime}$, i.e. $\mathscr{V}_{g} \in \mathfrak{F}\left(\mathcal{O}_{2}\right)$; moreover, by Eq. (2.2) we have for each $F \in \mathfrak{F}\left(\mathcal{O}_{1}\right) \vee \mathfrak{F}\left(\mathcal{O}_{2}\right)^{\prime}, h, g \in \mathscr{G}$,

$$
\begin{aligned}
\alpha_{h}\left(\mathscr{V}_{g}\right) F \xi & =\mathscr{U}(h) \mathscr{V}_{g} \mathscr{U}(h)^{-1} F \xi \\
& =\alpha_{h} \alpha_{g}^{1} \alpha_{h}^{-1}(F) \xi=\alpha_{h h^{-1}}^{1}(F) \xi \\
& =\mathscr{V}_{h h^{-1}} F \xi ;
\end{aligned}
$$

hence

$$
\alpha_{h}\left(\mathscr{V}_{g}\right)=\mathscr{V}_{h g h^{-1}}, \quad h, g \in \mathscr{G} .
$$

Denoting by $\mathscr{B}$ the von Neumann algebra generated by $\mathscr{V}_{g}, g \in \mathscr{G}$, and by $\mathscr{A}=\mathscr{B} \cap \mathscr{B}^{\prime}$ its center, we have from (2.5) that $\alpha_{g}(B)=\mathscr{V}_{g} B \mathscr{V}_{g}^{-1}$ for each $B \in \mathscr{B}$, hence $\mathscr{A} \subset \mathscr{U}(\mathscr{G})^{\prime} ;$ since $\mathscr{B} \subset \mathfrak{F}\left(\mathcal{O}_{2}\right), \mathscr{A} \subset \mathfrak{F}\left(\mathcal{O}_{2}\right) \cap \mathscr{U}(\mathscr{G})^{\prime}=\mathfrak{U}\left(\mathcal{O}_{2}\right)$; since $\mathscr{V}_{g}$ induces $\alpha_{g}$ on $\mathfrak{F}\left(\mathcal{O}_{1}\right)$, $\mathscr{B}$ commutes with $\mathfrak{A}\left(\mathcal{O}_{1}\right)$ and $\mathscr{A} \subset \mathfrak{A}\left(\mathcal{O}_{1}\right)^{\prime} \cap \mathfrak{U}\left(\mathcal{O}_{2}\right)$; as in [1] the observables in $\mathscr{A}$ can be interpreted as local measurements of the superselection quantum numbers.

We proved the following:

2.1. Theorem. Under the Assumptions (i)-(iii) there is a continuous unitary covariant local implementation of the gauge automorphisms in the sense of Eqs. (1.3) and (1.4).

A case which is important in elementary particle physics is when $\mathscr{G}$ is a Lie group. Let $\tilde{\mathscr{G}}_{0}$ be the simply connected covering of the connected component $\mathscr{G}_{0}$ of the identity in $\mathscr{G}, \sigma$ the canonical homomorphism of $\tilde{\mathscr{G}}_{0}$ into $\mathscr{G}$, and $\mathscr{L}$ the Lie algebra of $\mathscr{G}$. Denote by $[u, v], u, v \in \mathscr{L}$ the Lie product and by $u \in \mathscr{L} \rightarrow g(u) \in \mathscr{L}$ the automorphism of $\mathscr{L}$ determined by $g \in \mathscr{G}_{0} ; u \in \mathscr{L}$ acts on each $\mathbb{F}(\mathcal{O})$ as an unbounded derivation $\delta_{u}$ such that $\exp i \lambda \delta_{u}=\alpha_{\exp i \lambda u}$.

The infinitesimal generators of the representation of Theorem 2.1 provide a "local current algebra" associated to the pair $\mathcal{O}_{1}, \mathcal{O}_{2}$ in the sense that:

1. there is a linear map $u \in \mathscr{L} \rightarrow J_{u}$, with $J_{u}=J_{u}^{*}$ affiliated to $\mathfrak{F}\left(\mathcal{O}_{2}\right)$;

2. $\left[J_{u}, J_{v}\right]=i J_{[u, v]} ; \quad u, v \in \mathscr{L}$;

3. $\left[J_{u}, F\right]=\delta_{u}(F) ; \quad F \in \mathfrak{F}\left(\mathcal{O}_{1}\right), u \in \mathscr{L}$;

4. $\alpha_{g}\left(J_{u}\right)=J_{g(u)} ; \quad g \in \mathscr{G}_{0}, u \in \mathscr{L}$. 
More precisely, there should be a dense invariant common domain $\mathscr{D}$ of analytic vectors for each $J_{u}, u \in \mathscr{L}$ and a common core $\mathfrak{F}_{0}\left(\mathcal{O}_{1}\right) \subset \mathfrak{F}\left(\mathcal{O}_{1}\right)$ for each $\delta_{u}, u \in \mathscr{L}$, leaving $\mathscr{D}$ stable and 2.3 should hold for $F \in \mathfrak{F}_{0}\left(\mathcal{O}_{1}\right)$ and acting on vectors from $\mathscr{D}$.

The local current algebras as defined by (2.6) are precisely the generators of those continuous unitary representations $\tilde{\mathscr{V}}$ of $\mathscr{G}_{0}$ such that

(a) $\tilde{\mathscr{V}}_{g} \in \mathfrak{F}\left(\mathrm{O}_{2}\right), \quad g \in \tilde{\mathscr{G}}_{0}$;

(b) $\tilde{\mathscr{V}}_{g} F \tilde{\mathscr{V}}_{g}^{-1}=\alpha_{\sigma(g)}(F), \quad g \in \tilde{\mathscr{G}}_{0}, F \in \mathfrak{F}\left(\mathcal{O}_{1}\right)$;

(c) $\alpha_{\sigma(h)}\left(\tilde{\mathscr{V}}_{g}\right)=\tilde{\mathscr{V}}_{h h^{-1}}, \quad h, g \in \tilde{\mathscr{G}}_{0}$,

as follows from the general theory of Lie groups [8] (see appendix).

With $\mathscr{V}_{g}$ defined as in Eq. (2.4), note that $\mathscr{V}_{g}$ induces automorphisms of the relative commutant $\mathfrak{F}\left(\mathcal{O}_{1}\right)^{\prime} \cap \mathfrak{F}\left(\mathcal{O}_{2}\right)$; denote by $\tilde{\theta}$ the action of $\tilde{\mathscr{G}}_{0}$ on $\mathfrak{F}\left(\mathcal{O}_{1}\right)^{\prime} \cap \mathfrak{F}\left(\mathcal{O}_{2}\right)$ defined by

$$
\tilde{\theta}_{g}(F)=\mathscr{V}_{\sigma(g)} F \mathscr{V}_{\sigma(g)}^{-1}, \quad g \in \tilde{\mathscr{G}}_{0}, F \in \mathfrak{F}\left(\mathcal{O}_{1}\right)^{\prime} \cap \mathfrak{F}\left(\mathcal{O}_{2}\right)
$$

It is immediate that every continuous unitary representation $\tilde{\mathscr{V}}$ of $\tilde{\mathscr{G}}_{0}$ on $\mathscr{H}$ fulfilling (2.7) is given by

$$
\tilde{\mathscr{V}}_{g}=X_{g} \mathscr{V}_{\sigma(g)}, \quad g \in \tilde{\mathscr{G}}_{0},
$$

where $X_{g}$ is a covariant continuous unitary $\tilde{\theta}$-cocycle in $\mathfrak{F}\left(\mathcal{O}_{1}\right)^{\prime} \cap \mathfrak{F}\left(\mathcal{O}_{2}\right)$, i.e.

$$
\begin{gathered}
X_{g} \in \mathfrak{F}\left(\mathcal{O}_{1}\right)^{\prime} \cap \mathfrak{F}\left(\mathcal{O}_{2}\right) ; X_{g} \tilde{\theta}_{g}\left(X_{h}\right)=X_{g h}, \quad h, g \in \tilde{\mathscr{G}}_{0}, \\
\alpha_{\sigma(h)}\left(X_{g}\right)=X_{h g h^{-1}}, \quad h, g \in \tilde{\mathscr{G}}_{0} .
\end{gathered}
$$

Therefore we have

2.2. Theorem. The local current algebras associated with the pair $\mathcal{O}_{1} \ll \mathcal{O}_{2}$, $\mathcal{O}_{1}, \mathcal{O}_{2} \in \mathscr{K}$, in the sense of Eq.(2.6), are in one-to-one correspondence with the covariant continuous unitary $\tilde{\theta}$-cocycles in $\mathfrak{F}\left(\mathcal{O}_{1}\right)^{\prime} \cap \mathfrak{F}\left(\mathcal{O}_{2}\right)$ in the sense of Eq. (2.9).

We next compare the foregoing discussion with the usual current algebra hypothesis of particle physics ([16]; for further references and applications such as the famous Adler-Weissberger and Cabibbo-Radicati sum rules, see e.g. [17]). It is assumed there that to each element $u$ in the Lie algebra $\mathscr{L}$ of $\mathscr{G}$ is associated a Wightman vector field $j_{u}^{\mu}$, which is a conserved current ${ }^{1}$ whose $\mu=0$ component is a density for the generator of the representation of the gauge group associated to $u$, transforms covariantly under $\mathscr{G}_{0}$ and fulfills the equal time commutation rules

$$
\left[j_{u}^{0}(0, \mathbf{x}), j_{v}^{0}(0, \mathbf{y})\right]=i \delta(\mathbf{x}-\mathbf{y}) j_{[u, v]}^{0}(0, \mathbf{x}) .
$$

The relations (2.10) are clearly formal. In the most favourable case we can obtain (essentially) selfadjoint operators as the usual regularizations

$$
J_{u}=\int j_{u}^{0}(t, \mathbf{x}) f_{R}(\mathbf{x}) g_{\delta}(t) d^{3} x d t,
$$

where the test function is the convolution

$$
f_{R} g_{\delta}=\chi_{R} \delta * h
$$

1 Of course we have in mind currents associated with the exact internal symmetries of strong interactions 
of the product of the characteristic function of a sphere in space with the Dirac measure at $t=0$, with a non-negative function in $\mathscr{D}, h(x)=h_{1}(|\mathbf{x}|) g_{\delta}(t)$, with total integral 1.

With an appropriate choice of the test functions we can fulfill Eq. (2.6) 1,3,4, but it does not seem obvious how to satisfy point 2 . For that purpose we would need to remove the smoothing function $h$ from (2.12); unfortunately $J_{u}$ would be then a bilinear form and not an operator, already in the case of the free fields.

The existence of "local current algebras" does not seem to be a trivial consequence of the usual current algebra hypothesis. As the above discussion shows, it relies rather on the possibility of perturbing each $J_{u}$ in $(2.11)$ with a term affiliated to $\mathfrak{F}\left(\mathcal{O}_{1}\right)^{\prime} \cap \mathfrak{F}\left(\mathcal{O}_{2}\right)$ so that Eq. (2.6) holds.

Of course the converse problem would be of great interest: is it possible to deduce from the existence of local current algebras for each pair $\mathcal{O}_{1} \ll \mathcal{O}_{2}$, the existence of Wightman fields $j_{u}^{\mu}, u \in \mathscr{L}$, which are conserved currents locally associated with the field algebras $\mathfrak{F}(\mathcal{O})$, whose regularized time components fulfill relations $[(2.6), 1,3,4]$ [and possibly, in an appropriate limit, fulfill relations $(2.10)]$ ? It is clear that any progress on this problem is likely to require much more structure than that used in this note.

In particular the assumption of relativistic covariance of the theory is bound to be crucial. Here we limit ourselves to noticing that, under such an assumption, our local current algebras have a property of relativistic covariance.

Assume that there is a strongly continuous unitary representation $L \in \tilde{\mathscr{P}} \rightarrow \mathscr{U}(L)$ of the covering group $\tilde{\mathscr{P}}$ of the inhomogeneous Lorentz group acting on $\mathscr{H}$, such that for each double cone $\mathcal{O} \in \mathscr{K}$ and each gauge transformation $g \in \mathcal{O}$ we have

$$
\begin{aligned}
\mathscr{U}(L) \mathfrak{F}(\mathcal{O}) \mathscr{U}(L)^{-1} & =\mathfrak{F}(L \mathcal{O}), \\
\mathscr{U}(L) \mathscr{U}(g) & =\mathscr{U}(g) \mathscr{U}(L), \\
\mathscr{U}(L) \Omega & =\Omega, \quad L \in \tilde{\mathscr{P}} .
\end{aligned}
$$

Denote by $\mathscr{V}^{\left(\mathcal{O}_{1}, \mathfrak{O}_{2}\right)}$ the representation of $\mathscr{G}$ by unitaries in $\mathscr{F}\left(\mathcal{O}_{2}\right)$ canonically associated to the pair $\mathcal{O}_{1}, \mathcal{O}_{2} \in \mathscr{K}, \mathcal{O}_{1} \mathbb{E} \mathcal{O}_{2}$, defined by Eq. (2.4).

Using Lemma 3.3, by an argument similar to the one leading to Eq. (2.5), it is easily seen that for each pair $\left(\mathcal{O}_{1}, \mathcal{O}_{2}\right)$ and $L \in \tilde{\mathscr{P}}$ we have

$$
\mathscr{U}(L) \mathscr{V}^{\left(\mathcal{O}_{1}, \mathcal{O}_{2}\right)}(g) \mathscr{U}(L)^{-1}=\mathscr{V}^{\left(L \mathfrak{O}_{1}, L \mathcal{O}_{2}\right)}(g) .
$$

If $\mathscr{G}$ is a Lie group, let $J_{u}^{\left(\mathscr{O}_{1}, \mathcal{O}_{2}\right)}$ be the canonical current algebra associated to the pair $\mathcal{O}_{1}, \mathcal{O}_{2}$, obtained as the generators of the representation $\mathscr{V}^{\left(\mathcal{O}_{1}, \mathcal{O}_{2}\right)} \mid \mathscr{G}_{0}$. By Eq. (2.14) we have, for each pair $\mathcal{O}_{1}, \mathcal{O}_{2}$, and $L \in \tilde{\mathscr{P}}$,

$$
\mathscr{U}(L) J_{u}^{\left(\mathcal{O}_{1}, \mathcal{O}_{2}\right)} \mathscr{U}(L)^{-1}=J_{u}^{\left(L \mathcal{O}_{1}, L \mathcal{O}_{2}\right)}, \quad u \in \mathscr{L} .
$$

2.3. Theorem. In a relativistic theory fulfilling (i)-(iii) and (2.13), the canonical current algebras form a Poincaré covariant family; the Poincaré covariant choices of the local current algebras $\left(\mathcal{O}_{1}, \mathcal{O}_{2}\right) \rightarrow \tilde{J}_{u}^{\left(\mathcal{O}_{1}, \mathcal{O}_{2}\right)}, u \in \mathscr{L}$, are in one-to-one correspondence with the Poincaré covariant choices $\left(\mathbb{O}_{1}, \mathcal{O}_{2}\right) \rightarrow X_{g}^{\left(\mathscr{O}_{1}, O_{2}\right)}$ of gauge covariant, continuous unitary $\tilde{\theta}$-cocycles in $\mathfrak{F}\left(\mathcal{O}_{1}\right)^{\prime} \cap \mathfrak{F}\left(\mathcal{O}_{2}\right)$, i.e. fulfilling Eq. (2.9) and

$$
\mathscr{U}(L) X_{g}^{\left(\mathscr{O}_{1}, \mathcal{O}_{2}\right)} \mathscr{U}(L)^{-1}=X_{g}^{\left(L \mathscr{O}_{1}, L \mathcal{O}_{2}\right)}, \quad g \in \mathscr{G}, \quad L \in \tilde{\mathscr{P}} .
$$


Proof. Immediate by the discussion above and Theorem 2.2.

The field theoretical analogs (2.11) would fulfill relation (2.15), e.g. with the following choice. Let $\left(\mathcal{O}_{1}, \mathcal{O}_{2}\right)$ be double cones in the rest frame centered at the origin and let $f_{R} g$ be a test function as in Eq. (2.12), such that the operators (2.11) fulfill Eq. $[(2.6), 1,3,4]$ for the pair $\mathcal{O}_{1}, \mathcal{O}_{2}$; with $L \in \tilde{\mathscr{P}}, L x=\Lambda x+a, x \in \mathbb{R}^{4}$, let $\varepsilon=\Lambda(1,0,0,0)$ and define

$$
J_{u}^{\left(L \mathcal{O}_{1}, L \mathcal{O}_{2}\right)}=\int j_{u}^{v}(x) \varepsilon_{v} f_{R} g_{\delta}\left(\Lambda^{-1}(x-a)\right) d^{4} x, \quad u \in \mathscr{L}
$$

then Eqs. (2.15) and $[(2.6), 1,3,4]$ are fulfilled for $\left(L \mathcal{O}_{1}, L \mathcal{O}_{2}\right), L \in \tilde{\mathscr{P}}$.

Possible extensions of the present analysis concern the problem of derivation of sum rules (see e.g. [17]) from our local current algebras, and the problem of extending our method to symmetries which do not leave each local algebra stable, as e.g. the space time translations, or to models with supersymmetries.

\section{Standard and Split Inclusions of von Neumann Algebras}

Let $\mathfrak{A} \subset \mathfrak{B}$ be von Neumann algebras acting on a Hilbert space $\mathscr{H}$ and $\Omega$ a unit vector in $\mathscr{H}$.

3.1. Definition. $\{\mathfrak{U}, \mathfrak{B}, \Omega\}$ is a standard inclusion acting on $\mathscr{H}$ and $\Omega$ is a standard vector for $\mathfrak{A}, \mathfrak{B}$ if $\Omega$ is cyclic and separating for each of the algebras $\mathfrak{A}, \mathfrak{B}, \mathfrak{I}^{\prime} \cap \mathfrak{B}$. A standard inclusion is a triple $\{\mathfrak{A}, \mathfrak{B}, \omega\}$, where $\mathfrak{A} \subset \mathfrak{B}$ are von Neumann algebras and $\omega$ is a faithful normal state of $\mathfrak{B}$ such that the GNS representation of $\{\mathfrak{B}, \omega\}$ gives rise to a standard inclusion $\left\{\pi_{\omega}(\mathfrak{U}), \pi_{\omega}(\mathfrak{B}), \xi_{\omega}\right\}$ acting on $\mathscr{H}_{\omega}$; the latter will be called the GNS representation of the standard inclusion $\{\mathfrak{A}, \mathfrak{B}, \omega\}$.

3.2. Definition. A standard inclusion $\{\mathfrak{U}, \mathfrak{B}, \omega\}$ is split if there is a type I factor $\mathfrak{M}$ such that

\section{$\mathfrak{U} \subset \mathfrak{M} \subset \mathfrak{B}$.}

Standard and split inclusions are studied in detail in [7]; here we will limit ourself to discussing some basic facts for our application and to quote from [7] some results relevant to Field Theory. Note that Assumptions (i)-(iii) of Sect. 1 can be phrased by saying that if $\mathcal{O}_{1} \ll \mathcal{O}_{2}$ are double cones and $\omega_{0}$ is the vacuum state, $\left\{\mathfrak{F}\left(\mathcal{O}_{1}\right), \mathfrak{F}\left(\mathcal{O}_{2}\right), \omega_{0}\right\}$ is a standard split inclusion.

Let $\{\mathfrak{U}, \mathfrak{B}, \xi\}$ be a standard split inclusion acting on $\mathscr{H}$ and denote by $\omega$ the state of $\mathfrak{B}(\mathscr{H})$ induced by $\xi$. We can define a normal state $\varphi$ on the Neumann algebra $\mathfrak{R}=\mathfrak{A} \vee \mathfrak{B}^{\prime}$ by setting

$$
\varphi(A B)=\omega(A) \omega(B) ; \quad A \in \mathfrak{A}, \quad B \in \mathfrak{B}^{\prime} .
$$

Since $\mathfrak{R}$ is the commutant of $\mathfrak{U}^{\prime} \cap \mathfrak{B}, \xi$ is cyclic and separating for $\mathfrak{R}$ and each normal state of $\mathfrak{R}$ is induced by a unique vector in the cone

$$
\mathscr{P}_{\xi}^{\natural}(\mathfrak{R})=\overline{4^{1 / 4} \mathfrak{R}_{+} \xi}
$$

(see [9-11]), where $\Delta$ is the modular operator of $\mathfrak{R}$ defined by $\xi$. Moreover, a vector in the cone (3.2) is cyclic for $\mathfrak{R}$ iff it is separating for $\mathfrak{R}$. Let $\eta$ be the vector in the cone (3.2) inducing on $\mathfrak{R}$ the state $\varphi$ of Eq. (3.1). Since $\{\mathfrak{A}, \mathfrak{B}, \omega\}$ is split there is an isomorphism of $\mathfrak{R}$ onto $\mathfrak{A} \otimes \mathfrak{B}^{\prime}$ carrying $\varphi$ onto the state induced by the vector 
$\xi \otimes \xi[6]$. Since $\xi$ is cyclic for $\mathfrak{U}^{\prime}$ and $\mathfrak{B}, \xi \otimes \xi$ is cyclic for $\mathfrak{U}^{\prime} \otimes \mathfrak{B}$ and separating for $\mathfrak{U} \otimes \mathfrak{B}^{\prime}=\left(\mathfrak{U}^{\prime} \otimes \mathfrak{B}\right)^{\prime}[12]$, i.e. $\varphi$ is faithful and $\eta$ is cyclic and separating for $\mathfrak{R}$.

3.3. Lemma. The vector $\eta$ is covariantly associated to the triple $\{\mathfrak{A}, \mathfrak{B}, \xi\}$.

Proof. Let $\left\{\mathfrak{H}_{1}, \mathfrak{B}_{1}, \xi_{1}\right\}$ be a standard inclusion acting on $\mathscr{H}_{1}$ and $\eta_{1} \in \mathscr{H}_{1}$ the unit vector associated to it by the above procedure. With $U$ a unitary operator of $\mathscr{H}$ onto $\mathscr{H}_{1}$ such that

$$
U \mathfrak{A} U^{-1}=\mathfrak{A}_{1}, \quad U \mathfrak{B} U^{-1}=\mathfrak{B}_{1}, \quad U \xi=\xi_{1},
$$

we have to show that

$$
U \eta=\eta_{1} .
$$

Note that $U \mathfrak{R} U^{-1}=\mathfrak{A}_{1} \vee \mathfrak{B}_{1}^{\prime} \equiv \mathfrak{R}_{1}$ and by (3.3) the vector $U \eta$ induces on $\mathfrak{R}_{1}$ the state $\varphi_{1}$ defined by

$$
\begin{aligned}
& \varphi_{1}(A B)=\omega_{1}(A) \omega_{1}(B), \quad A \in \mathfrak{U}_{1}, \quad B \in \mathfrak{B}_{1}^{\prime}, \\
& \omega_{1}(C)=\left(\xi_{1}, C \xi_{1}\right), \quad C \in \mathfrak{B}\left(\mathscr{H}_{1}\right) \text {. }
\end{aligned}
$$

Hence $U \eta$ and $\eta_{1}$ induce the same normal state on $\mathfrak{R}_{1}$ and we have $U \eta=\eta_{1}$ if we prove that $U \eta \in \mathscr{P}_{\xi_{1}}^{\natural}\left(\Re_{1}\right)$. From the relations $U \mathfrak{R} U^{-1}=\mathfrak{R}_{1}$ and $U \xi=\xi_{1}$, and from the uniqueness of the modular operator we have that $U \Delta U^{-1}=\Delta_{1}$ is the modular operator of $\mathfrak{R}_{1}$ defined by $\xi_{1}$, and $U \mathscr{P}_{\xi}^{\natural}(\mathfrak{R})=\mathscr{P}_{\xi_{1}}^{\natural}\left(\mathfrak{R}_{1}\right)$; therefore $U \eta=\eta_{1}$ as desired.

With $\mathfrak{W}_{i}=\left\{\mathfrak{U}_{i}, \mathfrak{B}_{i}, \omega_{i}\right\}, \quad i=1,2$, two standard inclusions of von Neumann algebras, an isomorphism $\varrho$ of $\mathfrak{B}_{1}$ on $\mathfrak{B}_{2}$ is defined as an isomorphism $\varrho$ of $\mathfrak{B}_{1}$ onto $\mathfrak{B}_{2}$ such that $\varrho\left(\mathfrak{A}_{1}\right)=\mathfrak{A}_{2}, \omega_{2} \circ \varrho=\omega_{1}$.

3.4. Theorem. For each standard split inclusion of von Neumann algebras $\mathfrak{W}=\{\mathfrak{U}, \mathfrak{B}, \omega\}$, there is a canonical type I factor $\mathfrak{N}_{\mathfrak{W}}$ such that $\mathfrak{U} \subset \mathfrak{N}_{\mathfrak{B}} \subset \mathfrak{B} ; \mathfrak{N}_{\mathfrak{B}}$ is covariantly associated to $\mathfrak{W}$, i.e. if $\varrho$ is an isomorphism of $\mathfrak{B}_{1}$ on $\mathfrak{W}_{2}$,

$$
\varrho\left(\mathfrak{N}_{\mathfrak{W}_{1}}\right)=\mathfrak{N}_{\mathfrak{B}_{2}} \text {. }
$$

Proof. Let $\{\mathfrak{U}, \mathfrak{B}, \xi\}$ be the GNS representation of $\mathfrak{B}_{1}$ identifying $\mathfrak{A}$ and $\mathfrak{B}$ with their images acting on $\mathscr{H}$. Let $\eta \in \mathscr{H}$ be the vector associated to $\{\mathfrak{H}, \mathfrak{B}, \xi\}$ as in Lemma 3.3, inducing the product state $\varphi$, Eq. (3.1), on $\mathfrak{A} \vee \mathfrak{B}^{\prime}$. Let $E_{\eta}$ be the cyclic projection in $\mathfrak{B}$ defined by $\eta$, i.e.

$$
E_{\eta} \mathscr{H}=\overline{\mathfrak{B}^{\prime} \eta}
$$

We define $\mathfrak{R}_{\mathfrak{B}}$ as the von Neumann algebra generated by $\mathfrak{A}$ and $E_{\eta}$; than $\mathfrak{U} \subset \mathfrak{N}_{\mathfrak{B}} \subset \mathfrak{B}$ and $\mathfrak{N}_{\mathfrak{W}}$ is a type I factor [4].

With $\varrho$ an isomorphisms of $\mathfrak{M}_{1}$ on $\mathfrak{M}_{2}, \mathfrak{M}_{i}=\left\{\mathfrak{U}_{i}, \mathfrak{B}_{i}, \omega_{i}\right\}$ standard split inclusions with GNS representations $\left\{\mathfrak{A}_{i}, \mathfrak{B}_{i}, \xi_{i}\right\}$ on $\mathscr{H}_{i}$, we can define a unitary operator of $\mathscr{H}_{1}$ on $\mathscr{H}_{2}$ by

$$
U B \xi_{1}=\varrho(B) \xi_{2}, \quad B \in \mathfrak{B}_{1} .
$$

By Eq. (3.7) we have $U \mathfrak{A}_{1} U^{-1}=\mathfrak{A}_{2}, U \mathfrak{B}_{1} U^{-1}=\mathfrak{B}_{2}, U \xi_{1}=\xi_{2}$. With $\eta_{i} \in \mathscr{H}_{i}$ the vector associated as above to the triple $\left\{\mathfrak{U}_{i}, \mathfrak{B}_{i}, \xi_{i}\right\}, i=1,2$, we have by Lemma 3.3

$$
U \eta_{1}=\eta_{2} \text {. }
$$


Since $U \mathfrak{B}_{1}^{\prime} U^{-1}=\mathfrak{B}_{2}^{\prime}$ by (3.6) and (3.7) we have $U E_{\eta_{1}} U^{-1}=E_{\eta_{2}}$ and $U \mathfrak{N}_{\mathfrak{W}_{1}} U^{-1}=\varrho\left(\mathfrak{N}_{\mathfrak{W}_{1}}\right)=\mathfrak{N}_{\mathfrak{M}_{2}}$.

As a consequence of Theorem 3.4, if $\mathfrak{B}=\{\mathfrak{U}, \mathfrak{B}, \omega\}$ is a standard split inclusion and Aut $\mathfrak{B} \subset$ Aut $\mathfrak{B}$ is the group of automorphisms of $\mathfrak{M}$, then $\mathfrak{N}_{\mathfrak{B}}$ is globally invariant under Aut $\mathfrak{B}$. As shown in [7], it follows that Aut $\mathfrak{B}$ is a compact metrizable group with the topology of strong convergence on the predual of $\mathfrak{B}$. Moreover, in the unitary representation of Aut $\mathfrak{B}$ induced by $\omega$, each finite dimensional representation of Aut $\mathfrak{B}$ appears and has infinite multiplicity.

\section{Semilocal Nets of Type I Factors}

Let $\mathcal{O} \in \mathscr{K} \rightarrow \mathfrak{U}(\mathcal{O})$ be the net of algebras of local observables acting on the vacuum Hilbert space $\mathscr{H}_{0}$ of a local quantum theory fulfilling additivity, relativistic invariance, spectrum condition and duality. If $\mathcal{O}_{1} \leqslant \mathcal{O}_{2}, \mathcal{O}_{1}, \mathcal{O}_{2} \in \mathscr{K}$, by the ReehSchlieder theorem the vacuum state vector $\Omega$ is cyclic and separating for $\mathfrak{A}\left(\mathcal{O}_{1}\right)$, $\mathfrak{U}\left(\mathcal{O}_{2}\right)$, and $\mathfrak{A}\left(\mathcal{O}_{1}\right)^{\prime} \cap \mathfrak{U}\left(\mathcal{O}_{2}\right)$, i.e. $\left\{\mathfrak{U}\left(\mathcal{O}_{1}\right), \mathfrak{U}\left(\mathcal{O}_{2}\right), \omega_{0}\right\}$ is a standard inclusion that we assume here is also split.

Let $I \subset \mathscr{K} \times \mathscr{K}$ be the set of pairs $w=\left(\mathcal{O}_{w}, \tilde{\mathcal{O}}_{w}\right)$ of double cones such that $\mathcal{O}_{w} \ll \tilde{\mathcal{O}}_{w}$; we will write $w_{1} \subset w_{2}$ if $\tilde{\mathcal{O}}_{w_{1}} \subset \mathcal{O}_{w_{2}}, w_{1} \sim w_{2}$ if $\tilde{\mathcal{O}}_{w_{1}} \subset \tilde{\mathcal{O}}_{w_{2}}^{\prime}$, and $L w=\left(L \mathcal{O}_{w}, L \mathcal{O}_{w}\right)$ with $L$ a Poincaré transformation.

Denote by $\mathscr{U}_{0}$ the unitary continuous representation implementing the Poincaré transformations on $\mathfrak{A}$.

To each $w \in I$ we associate the canonical type I factor $\mathfrak{N}_{w}$ determined by the standard split inclusion $\left\{\mathfrak{U}\left(\mathcal{O}_{w}\right), \mathfrak{U}\left(\tilde{\mathcal{O}}_{w}\right), \omega_{0}\right\}$.

4.1. Theorem. The net $\mathfrak{N}_{w}$, weI fulfills the following properties:

1. $\mathfrak{N}_{w_{1}} \subset \mathfrak{N}_{w_{2}}$ if $w_{1} \subset w_{2}$,

2. $\mathfrak{N}_{w_{1}} \subset \mathfrak{M}_{w_{2}}^{\prime}$ if $w_{1} \sim w_{2}$,

3. $\mathscr{U}_{0}(L) \mathfrak{N}_{w} \mathscr{U}_{0}(L)^{-1}=\mathfrak{N}_{L w}, \quad L \in \mathscr{P}$,

4. $\left\{\mathfrak{N}_{w_{1}} / w_{1} \in I, \tilde{\mathcal{O}}_{w_{1}} \subset \mathcal{O}^{\prime}\right\}^{\prime \prime}=\left\{\mathfrak{N}_{w_{2}}^{\prime} / w_{2} \in I, \mathcal{O} \subset \mathcal{O}_{w_{2}}\right\}^{\prime \prime}, \quad \mathcal{O} \in \mathscr{K}$,

5. $\tilde{\mathcal{O}}_{w} \subset \mathcal{O}_{w_{1}} \cup \ldots \cup \mathcal{O}_{w_{n}} \Rightarrow \mathfrak{N}_{w} \subset \mathfrak{N}_{w_{1}} \vee \ldots \vee \mathfrak{N}_{w_{n}}, \quad w, w_{1}, w_{n} \in I$.

Proof. All properties are trivial consequences of the definition and of Theorem 3.4, or of the relation

$$
\mathfrak{U}(\mathcal{O})=\bigcap\left\{\mathfrak{N}_{w} / w \in I, \mathcal{O} \subset \mathcal{O}_{w}\right\}, \quad \mathcal{O} \in \mathscr{K},
$$

which follows from the remark that, by duality,

$$
\mathfrak{U}(\mathcal{O})=\bigcap\left\{\mathfrak{U}\left(\mathcal{O}_{\lambda}\right) / \mathcal{O}_{\lambda} \in \mathscr{K}, \mathcal{O} \ll \mathcal{O}_{\lambda}\right\} .
$$

A net $\mathfrak{N}_{w}, w \in I$ of type I factors acting on a Hilbert space $\mathscr{H}_{0}$ will be called a semilocal net if it fulfills 1 and 2 in Theorem 4.1 and we will call properties 3-5 respectively relativistic invariance, duality, additivity of the semilocal net. 
4.2. Theorem. Given a semilocal net of type I factors $\mathfrak{N}_{w}, w \in I$ we can associate to it a local net $\mathfrak{R}(\mathcal{O}), \mathcal{O} \in \mathscr{K}$ by Eq. (4.1) which will fulfill relativistic invariance, additivity or duality if the semilocal net does.

\section{Proof. Immediate.}

The semilocal net defined in Theorem 4.1 may be called the canonical one and it may be easily seen that there are other semilocal nets which give back the original net by Eq. (4.1); it suffices to define $\mathfrak{M}_{w}=\mathfrak{M}_{f(w)}$ with $f: I \rightarrow I$ covariant and such that $\mathcal{O}_{w} \subset \mathcal{O}_{f(w)} \subset \tilde{\mathcal{O}}_{f(w)} \subset \tilde{\mathcal{O}}_{w}, w \in I$. The canonical semilocal net can play a role in structural questions concerning the algebra of quasilocal observables. One such question is whether there is a natural choice of a separable $C^{*}$-algebra $\mathfrak{B}$ dense in $\mathfrak{U}$ in the topology of each locally normal representation, or $\mathscr{T}$-dense in the sense of [1] (see also [13]). Under our present assumptions it is possible to construct the $C^{*}$-algebra $\mathfrak{B}$ by using the ideal of compact operators in $\mathfrak{N}_{w}$ for a dense sequence of $w$ 's and regularizing over the Poincaré group; then one defines $\mathfrak{B}(\mathcal{O})$ as $\mathfrak{U}(\mathcal{O}) \wedge \mathfrak{B}, \mathcal{O} \in \mathscr{K}$. This construction depends upon the choice of a dense sequence in $\mathbb{R}^{4}$. In a dilatation invariant theory, the initial choice would reduce to that of a single pair $w \in I$.

\section{Appendix 1}

We make here more explicit the derivation of formulas (2.7) from (2.6). First of all the representation $u \rightarrow J_{u}$ of $\mathscr{L}$ arises by differentiating a unique unitary representation $g \in \tilde{\mathscr{G}}_{0} \rightarrow \tilde{v}_{g} \in \mathfrak{F}_{\left(\mathcal{O}_{2}\right)}$ in (a) because of the existence of sufficiently many analytic vectors [14]. Formula (c) clearly follows from 4 of (2.6). Finally 3 of (2.6) implies (b) of (2.7) by the following argument.

Let $\delta_{u}^{\prime}$ be the generator of the one-parameter automorphism group of $\mathfrak{B}(\mathscr{H})$ implemented by $\exp \left(i J_{u} t\right)$; then $\mathfrak{F}_{0}\left(\mathcal{O}_{1}\right)$ is contained in the domain $\mathscr{D}\left(\delta_{u}^{\prime}\right)$ of $\delta_{u}^{\prime}[15]$ and $\delta_{u}^{\prime}(F)=\delta_{u}(F), F \in \mathfrak{F}_{0}\left(\mathcal{O}_{1}\right)$. Since $\delta_{u}$ and $\delta_{u}^{\prime}$ are both closed operators and $\mathfrak{F}_{0}\left(\mathcal{O}_{1}\right)$ is a core for $\delta_{u}$ [as a derivation of $\left.\mathscr{F}\left(\mathcal{O}_{1}\right)\right]$, we have $\mathscr{D}\left(\delta_{u}\right) \cap \mathfrak{F}\left(\mathcal{O}_{1}\right) \subset \mathscr{D}\left(\delta_{u}^{\prime}\right)$ and

$$
\delta_{u}^{\prime}(F)=\delta_{u}(F), \quad F \in \mathscr{D}\left(\delta_{u}\right) \cap \mathfrak{F}\left(\mathcal{O}_{1}\right)
$$

Let $F \in \mathscr{D}\left(\delta_{u}\right) \cap \mathfrak{F}\left(\mathcal{O}_{1}\right)$ be an analytic element for $\delta_{u}$, then (A.1) implies

$$
e^{i \lambda J_{u}} F e^{-i \lambda J_{u}}=\exp \left(i \lambda \delta_{u}^{\prime}\right)(F)=\exp \left(i \lambda \delta_{u}\right)(F)=\alpha_{\exp (i \lambda u)}(F)
$$

Since $\mathfrak{F}\left(\mathcal{O}_{1}\right)$ is invariant for $\alpha$, analytic elements in $\mathfrak{F}\left(\mathcal{O}_{1}\right)$ are dense in $\mathfrak{F}\left(\mathcal{O}_{1}\right)$, hence (A.2) holds for all $F \in \mathfrak{F}\left(\mathcal{O}_{1}\right)$. Therefore (c) holds for all $g$ in a one-parameter subgroup of $\tilde{\mathscr{G}}_{0}$, hence for all $g \in \tilde{\mathscr{G}}_{0}$.

Note added in proof. By work in progress of D. Buchholz [18] assumption (iii) Sect. 1 can be derived from a variant of the Haag-Swieca compactness criterion, expressing a local property related to asymptotic completeness and to natural restrictions on the particle spectrum. Therefore interesting theories can be described by semilocal nets of type I factors. On the other hand by [7] assumption (iii) excludes undesirable models as most of the generalized free fields or the tensor products of infinitely many copies of any given theory with itself. For further consequences see [7]. 


\section{References}

1. Doplicher, S.: Local aspects of superselection rules. Commun. Math. Phys. 85, 73 (1982)

2. Doplicher, S., Haag, R., Roberts, J. E. : Local observables and particle statistics, I. Commun. Math. Phys. 23, 198 (1971); II. Commun. Math. Phys. 35, 49 (1974)

3. Buchholz, D., Fredenhagen, K.: Locality and the structure of particle states. Commun. Math. Phys. 84, 1 (1982)

4. Buchholz, D.: Product states for local algebras. Commun. Math. Phys. 36, 287 (1974)

5. Fröhlich, J. : Quantum theory of nonlinear invariant wave equations. In : Invariant wave equations. Lecture Notes in Physics, Vol. 73. Velo, G., Wightman, A. S. (eds.). Berlin, Heidelberg, New York: Springer 1978

6. D'Antoni, C., Longo, R. : Interpolation by type I factors and the flip automorphism. J. Funct. Anal. (to appear)

7. Doplicher, S., Longo, R.: Standard and split inclusions of von Neumann algebras (preprint)

8. Cohn, P. M.: Lie groups. Cambridge: Cambridge University Press 1961

Chevalley, C.: Theory of Lie groups. Princeton, NJ : Princeton University Press 1946

9. Connes, A.: Caractérisation des espaces vectoriels ordonnés sous-jacents aux algèbres de von Neumann. Ann. Inst. Fourier, Grenoble 24, 121 (1974)

10. Araki, H. : Positive cones, .... Proceedings of the Int. School E. Fermi, Course LX, Kastler, D. (ed.). Soc. Italiana di Fisica. Amsterdam: North-Holland 1976; Recent developments in the theory of operator algebras. Symposia Mathematica XX, Istituto Nazionale di Alta Matematica. New York: Academic Press 1976

11. Haagerup, U.: The standard form of a von Neumann algebra. Math. Scand. 37, 271 (1975)

12. Takesaki, M.: Tomita's theory of modular Hilbert algebras. In: Lecture Notes in Mathematics, Vol. 128. Berlin, Heidelberg, New York: Springer 1970

13. Haag, R., Kadison, R. V., Kastler, D.: Nets of $C^{*}$-algebra and classification of states. Commun. Math. Phys. 16, 11 (1970)

14. Nelson, E.: Analytic vectors. Ann. Math. 70, 572 (1959); see also,

Simon, J.: On the integrability of representations of finite dimensional real Lie algebras. Commun. Math. Phys. 28, 39 (1972)

15. Bratteli, O., Robinson, D. W.: Unbounded derivations of von Neumann algebras. Ann. IHP 25, 139 (1976)

16. Gell-Mann, M.: The symmetry group of vector and axial vector currents. Physics 1, 63 (1964)

17. Treiman, S. B., Jackiw, R., Gross, D. J.: Lectures on current algebra and its applications. Princeton: Princeton University Press 1972

18. Buchholz, D.: in preparation (private communication).

Communicated by R. Haag

Received October 5, 1982 
